\title{
Healthcare disparities among octogenarians and nonagenarians with stage III lung cancer
}

\author{
Richard J. Cassidy, MD ${ }^{1}$, Xinyan Zhang, MPH ${ }^{2}$, Jeffrey M. Switchenko, PhD ${ }^{2}$, Pretesh R. \\ Patel, MD ${ }^{1}$, Joseph W. Shelton, MD ${ }^{1}$, Sibo Tian, MD' ${ }^{1}$, Ronica H. Nanda, MD ${ }^{3}$, Conor E. \\ Steuer, MD ${ }^{4}$, Rathi N. Pillai, MD ${ }^{4}$, Taofeek K. Owonikoko, MD, PhD ${ }^{4}$, Suresh S. Ramalingam, \\ MD ${ }^{4}$, Felix G. Fernandez, MD ${ }^{5}$, Seth D. Force, MD $^{5}$, Theresa W. Gillespie, PhD $^{6}$, Walter J. \\ Curran, $\mathbf{M D}^{1}$, and Kristin A. Higgins, $\mathbf{M D}^{1}$ \\ ${ }^{1}$ Department of Radiation Oncology, Winship Cancer Institute of Emory University, Atlanta, GA \\ ${ }^{2}$ Department of Biostatistics and Bioinformatics, Winship Cancer Institute of Emory University, \\ Atlanta, GA \\ ${ }^{3}$ University of Florida Proton Therapy Institute, Jacksonville, FL \\ ${ }^{4}$ Department of Medical Oncology, Winship Cancer Institute of Emory University, Atlanta, GA \\ ${ }^{5}$ Department of Thoracic Surgery, Winship Cancer Institute of Emory University, Atlanta, GA \\ ${ }^{6}$ Department of Surgery, Winship Cancer Institute of Emory University, Atlanta, GA
}

\section{Abstract}

Background-The practice patterns for patients $\geq 80$ years old with stage III non-small cell lung cancer (NSCLC) is not well known. The purpose of this study was to investigate factors predictive of and the impact on overall survival (OS) following concurrent chemoradiation (CRT) among patients $\geq 80$ years old with stage III NSCLC in the National Cancer Data Base (NCDB).

Methods-In the NCDB, patients $\geq 80$ years old with stage III NSCLC from 2004 to 2013 with complete treatment records were identified. Multivariable logistic regression and Cox-proportional hazard models were generated and propensity-score matched (PSM) analysis was employed.

Results-A total of 12,641 patients met entry criteria: 6,018 (47.6\%) were stage IIIA, 6,623 $(52.4 \%)$ were stage IIIB. Median age at diagnosis was 83.0 years (80-91). A total of 7,921 patients $(62.7 \%)$ received no therapy. Black race $(\mathrm{OR}=1.23$, 95\%CI: $1.06-1.43)$ and living in a lower-educated census-tract $(\mathrm{OR}=1.20,95 \% \mathrm{CI}: 1.03-1.40)$ were associated with not receiving care while treatment at an academic center $(\mathrm{OR}=0.80,95 \% \mathrm{CI}$ : 0.70-0.92) was associated with receiving cancer-directed therapy. Receipt of no treatment (HR=2.69, 95\%CI: $2.57-2.82)$ or

Corresponding Author: Richard J. Cassidy, MD, Radiation Oncology, Winship Cancer Institute of Emory University, Emory University School of Medicine, 1365 Clifton Road NE, Atlanta, GA 30322, richardjcassidy@ gmail.com Cell: 904-790-9207 Fax: 1-888-971-2304.

Author Contributions: RJC (all aspects), XZ (data collection, statistical analysis), PRP (planning, review), JWS (planning, review), ST (planning, review), RHN (planning, review), CES (planning, review), RNP (planning, review), TKO (planning, review), SSR (planning, review), FGF (planning, review), SDF (planning, review), TWG (planning, data collection, editing, review), WJC (planning, review), KAH (all aspects)

Conflicts of Interest/Disclosures: None 
definitive radiation alone $(\mathrm{HR}=1.15,95 \% \mathrm{CI}$ : $1.07-1.24)$ compared to CRT was associated with worse OS. On PSM not receiving CRT was associated with worse OS (HR=1.58, 95\%CI: 1.441.72).

Conclusions-In this NCDB analysis, $62.7 \%$ of patients 280 years old with stage III NSCLC received no cancer-directed care. Black race and living in a lower-educated census-tract were associated with no cancer-directed care. OS was improved in patients receiving CRT.

\section{Keywords}

healthcare disparities; lung cancer; National Cancer Data Base; radiation; chemotherapy; geriatrics; education; socioeconomic issues; race

\section{INTRODUCTION}

Lung cancer is the most common cancer cause of death in the United States (US), with over 158,000 patients dying annually ${ }^{1}$. The standard of care therapy for stage III non-small cell lung cancer is concurrent chemotherapy and radiation therapy (CRT), established in randomized clinical trials ${ }^{2-4}$. In the clinical trial setting, the median overall survival (OS) for patients with stage III non-small cell lung cancer approaches 29 months ${ }^{5}$. There is a lack of data for the use of CRT in octogenarians and nonagenarians, despite over 36\% of new lung cancers being diagnosed in patients $\geq 75$ years old ${ }^{1}$. These elderly patients may be undertreated and are routinely underrepresented on clinical trials for many reasons including concerns of medical fragility, lower patient willingness to pursue aggressive therapy, or concerns about the utility of therapy in patients that may die of competing risk factors ${ }^{6,7}$.

Highlighting the lack of clarity, recent guidelines for the management of elderly patients with stage III lung cancer state that "chemoradiation, either sequentially or concurrently, can be considered an option in elderly patients although it should be only used in selected, fit patients. Data are limited in the elderly and should be considered with caution." 8 Given the rising number of cancer patients 80 years and older ${ }^{9}$ and the lack of information about utility of CRT in this older population, we used the National Cancer Data Base (NCDB) to identify a large cohort of octogenarians and nonagenarians diagnosed with stage III non-small cell lung cancer. Our main goal was to identify patient and tumor factors associated with patients not receiving treatment and patients receiving CRT. Our secondary objective was to determine the impact of type of treatment received on OS.

\section{METHODS}

\section{Patient Selection}

The NCDB captures approximately $70 \%$ of newly diagnosed malignancies in the US. It only includes cases diagnosed at Commission on Cancer accredited facilities, a program of the American College of Surgeons that recognizes centers for providing comprehensive, highquality, and multidisciplinary patient centered care. This database includes detailed demographic, socioeconomic, disease, and treatment characteristics in addition to survival outcomes. 
The non-small cell lung NCDB was queried for patients diagnosed between 2004 and 2014. Our inclusion criteria included only patients that were at least 80 years old at diagnosis, have invasive histology, and have no history of a previous cancer diagnosis. Patients with stage I or II disease, metastatic disease, or with incomplete treatment or survival records were excluded (Supplementary Figure 1). We focused on stage III patients to homogenize our population and focus specifically on the impact of concurrent chemoradiation with survival. Our final population was classified into clinical stage IIIA or IIIB using modern criteria ${ }^{10}$. Treatment groups were no treatment, definitive radiation alone (defined as a minimum of 45 Gy to a thoracic volume), and CRT standard of care. The following patient characteristics were examined: age, sex, race (White, Black, and other), co-morbidities as quantified by the Charlson-Deyo Score ${ }^{11,12}$, insurance (Medicare, private, not insured), county of residence (urban, rural, or metro as defined by the US Census Bureau), percentage of residents without a high school degree in patient's census tract $(<7,7-12.9,13-20$, and $\geq 21 \%$ quartiles), and median income of patient's census tract $(<38,000,38,000-47,999,48,000-62,999$, and $>63,000$ dollars as determined by the American Community Survey). Census tracts are permanent subdivisions of a county that are updated by local participants prior to each national census, containing populations between 1,200 and 8,000 people. The following tumor characteristics were examined: histology (squamous cell carcinoma, adenocarcinoma, or biopsy proven but not otherwise specified), grade (well/moderately differentiated and poorly differentiated), clinical $\mathrm{T}$ stage, clinical $\mathrm{N}$ stage, and overall clinical stage (IIIA vs. IIIB). The following treatment characteristics were examined: facility location (northeast, south, midwest, and west) and facility type (academic, comprehensive community cancer center, and community cancer center). Age was evaluated as continuous variables after it was determined it had a linear effect on OS.

\section{Statistical Methods}

Statistical analysis was conducted using SAS Version 9.4 (Cary, NC) and SAS macros ${ }^{13}$. Descriptive statistics for each variable were reported and compared across treatment groups using Chi-Square and ANOVA tests where appropriate. Statistical models were described as follows:

Treatment Models-The response variables were: 1) receipt of no treatment versus (vs.) receipt of radiation alone or CRT; and 2) receipt of CRT vs. all others (no treatment or radiation alone). Explanatory variables included race, sex, insurance status, income, education, Charlson-Deyo comorbidity score, histology, tumor grade, tumor stage, facility location, and facility type. Univariate logistics regression models were built for each explanatory variable. Multivariable models were built allowing the explanatory variables to remain in the model using backwards elimination with a p-value for removal of 0.1. Additionally, a multinomial logistic regression model was run, comparing the three treatment groups and their association with patient, tumor, and treatment variables.

Overall Survival (OS) Models-The response variable was OS, defined as months from diagnosis to death or last follow up, where patients who did not die were censored at last follow-up. Explanatory variables included race, sex, insurance status, education, CharlsonDeyo score, histology, tumor grade, tumor stage, treatment type, facility location, and 
facility type. Univariate Cox proportional hazard models were built for each explanatory variable. Multivariable models were built by fixing treatment into each model, and allowing the other explanatory variables to remain in the model using backwards elimination with a pvalue for removal of 0.1. Kaplan-Meier (KM) curves were generated for OS stratified by treatment group, with comparisons made using log-rank tests. Two separate Coxproportional hazard models and KM OS analyses were run: one including all patients and one in which patients who died within 6 months from diagnosis were excluded. This analysis was done to reduce biases of the very ill who would be less likely to receive definitive therapy. Finally, propensity score matching was implemented to reduce treatment selection bias. A logistic regression model predicting for CRT versus other treatments was carried out to estimate the propensity score of all covariates. Patients were then matched 1:1 based on propensity score using a greedy 5-1-digit match algorithm ${ }^{14}$. After matching, the balance of the two groups was evaluated by standardized differences with values $<0.1$ considered negligible ${ }^{15}$. The OS effect in the matched sample was estimated using a Cox model with a robust variance estimator ${ }^{16,17}$.

The collinearity of among all variables was checked by removing variables with variance inflation factors greater than ten; however, no variables included in the multivariable analyses were too highly correlated. For each Cox survival model, the proportional hazard assumption was assessed by plotting the logarithms of the cumulative baseline hazard rate for each level of each covariate after adjusting for the effects of other variables. Timedependent covariates were also considered by multiplying the log survival time by each covariate. A time-dependent p-value greater than 0.05 suggested a valid proportional hazards assumption. For all analyses, a $\mathrm{p}<0.05$ was considered statistically significant.

\section{RESULTS}

\section{Patient Characteristics}

A total of 12,641 patients met study entry criteria (Supplementary Figure 1); 6,018 (47.6\%) were stage IIIA and 6,623 (52.4\%) were stage IIIB. The median age at diagnosis was 83.0 years (range 80.0 to 91.0 years) with a median follow up time of 28.6 months (range 2.1 to 140.7 months). The median radiation dose was $59.4 \mathrm{~Gy}$ (range $50.0 \mathrm{~Gy}$ to $71.0 \mathrm{~Gy}$ ). Supplementary Table 1 summarizes the remaining characteristics of our population.

\section{Predictors of Receiving No Treatment}

In total, 7,921 patients (62.7\%) received no therapy. On univariable analysis, older age, nonCaucasian race, treatment in the western United States, female sex, living in a lower educated census tract, adenocarcinoma histology, and stage IIIB tumors were more likely to not receive therapy while evaluation at an academic center was associated with receiving therapy (Table 1). On multivariable analysis, older age, non-Caucasian race, living in a lower-educated county, adenocarcinoma histology, poorly differentiated tumors, and stage IIIB tumors were associated with not receiving therapy while treatment at an academic center, male sex, and patients with a Charlson-Deyo score of 0 were more likely to receive therapy (Table 1). 


\section{Predictors of Receiving Concurrent Chemoradiation}

In total, 3,567 patients (28.2\%) received CRT. On univariable analysis, older age, male sex, Charlson-Deyo score of 0, living in an urban county, non-adenocarcinoma histology, and treatment at a facility located in the non-Western United States were associated with receipt of CRT while non-Caucasian race and stage IIIB tumors were associated with lack of receipt of CRT (Table 2). On multivariable analysis, male sex, Charlson-Deyo score of 0, living in an urban county, non-adenocarcinoma histology, and treatment at a facility located in the non-Western United States were associated with receipt of CRT. In the same model, advancing age, black race, living in a lower-educated county, and stage IIIB tumors were associated with lack of receipt of CRT (Table 2). Multinomial logistic regression model results for variables associated with receipt of different therapies are reported in Supplementary Table 2, with age, race, sex, insurance status, Charlson-Deyo score, tumor grade, tumor histology, tumor stage, facility location, facility type all significant (all $\mathrm{p}<0.01)$.

\section{Overall Survival}

Among all patients, two-year OS estimates were 31.0\% (95\%CI: 29.4-32.5\%) in the CRT cohort compared to $23.6 \%$ (95\%CI: $21.1-26.1 \%$ ) and $6.9 \%$ (95\%CI: $6.3-7.5 \%$ ) in the definitive radiation alone and no treatment cohorts, respectively $(\mathrm{p}<0.01$, Figure 1$)$. Among patients who survived at least six months from diagnosis, two-year OS estimates were $32.0 \%$ (95\%CI: 30.2-33.8\%) in the CRT cohort compared to $24.4 \%$ (95\%CI: $21.5-27.4 \%$ ) and $17.7 \%$ (95\% CI: $16.1-19.4 \%$ ) in the definitive radiation alone and no treatment cohorts, respectively ( $<<0.01$, Figure 2$)$.

On univariable analysis of all patients, receiving no treatment or definitive radiation alone (compared to CRT), older age, male sex, non-adenocarcinoma histology, stage IIIB tumors were associated with worse OS while Charlson-Deyo score of 0 , well differentiated tumors, and treatment at an academic facility were associated with improved OS (Table 3). On multivariable analysis of all patients, receipt of no treatment or definitive radiation alone compared to CRT was associated with worse OS. In the same model, advancing age, male sex, non-adenocarcinoma histology, and stage IIIB tumors were associated with worse OS while patients with a Charlson-Deyo score of 0 , well differentiated tumors, and treatment at an academic program were associated with improved OS (Table 3). Supplementary Table 3 details univariable and multivariable analyses of overall survival among patients who survived at least six months from diagnosis.

After propensity-score matching for all baseline variables, there were 1,153 patients in the CRT group matched to 1,153 patients who did not receive CRT. In this analysis, not receiving CRT was associated with worse OS (HR=1.58, 95\%CI: 1.44-1.72) on Cox proportional hazard analysis. Two-year OS estimates were 33.4\% (95\%CI: 30.3-36.5\%) in the CRT cohort versus $25.2 \%$ (95\% CI: 22.4-28.1\%) in the matched cohort ( $<<0.01$, Figure $3)$. 


\section{DISCUSSION}

Our series is the largest in the literature examining octogenarians and nonagenarians with stage III non-small cell lung cancer. Even in this older population, deviation from the standard of care of CRT was associated with worse OS. This OS benefit was confirmed when balancing for other demographic, socioeconomic, and disease variables, suggesting that the value of CRT is independent of age, race, co-morbidity status, histology, and treatment facility characteristics in this NCDB series. Alarmingly more than half of octoand nonagenarians with stage III lung cancer did not receive any care. Older age, black race, and living in a lower-educated census tract were risk factors for not getting any treatment. Conversely, males, treatment at an academic center, and improved comorbidity status were associated with receiving some form of therapy.

Patients 80 years and older are underrepresented on the landmark clinical trials that established CRT as the standard of care for stage III non-small cell lung cancer. RTOG 9410 was the landmark North American Phase III trial comparing two different concurrent chemoradiotherapy regimens with sequential chemotherapy followed by radiation ${ }^{2}$. Patients were randomized to concurrent cisplatin/vinblastine with $63 \mathrm{~Gy}$ of thoracic radiation given daily, cisplatin/vinblastine followed by $63 \mathrm{~Gy}$ of thoracic radiation at day 50 (sequential therapy), or cisplatin/etoposide with $69.6 \mathrm{~Gy}$ of thoracic radiation given twice daily. Fiveyear OS was improved in patients receiving concurrent cisplatin/vinblastine with radiation compared to sequential therapy, with rates of $16 \%$ and $10 \%$ respectively. OS was statistically improved in the concurrent cisplatin/vinblastine and radiation arm compared to the concurrent cisplatin/etoposide and twice daily radiation arm, with a hazard ratio of $0.93^{2}$. RTOG 9410 dramatically influenced practice and concurrent chemoradiation became the standard of care for medically-fit stage III lung cancer, however its applicability for octogenarians and nonagenarians is unclear. This trial did not have an upper limit cutoff for age enrollment, but closer examination of this trial shows a minimal number of patients 80 years and older. In fact, the maximum age of enrolled patients on the two vinblastine arms was 79 years old and the maximum age of enrolled patients on the etoposide arm was 80 years old. The limited enrollment of patients $\geq 80$ years old was also seen on a more recently published two-by-two factorial phase III trial, RTOG 0617. This trial investigated the efficacy of dose escalation of thoracic radiation of $60 \mathrm{~Gy}$ versus $74 \mathrm{~Gy}$ with concurrent carboplatin/paclitaxel chemotherapy ${ }^{5}$. This trial found that median OS was improved in patients receiving standard dose radiation compared to high-dose radiation, with rates of 28.7 months compared to 20.3 months respectively. This trial did accrue more octogenarians, with the eldest patient being 83 years' old. However, the median age of enrolled patients was 64 years old, suggesting that the number of octogenarians was a relatively small portion of this trial population ${ }^{5}$. The limited number of patients $\geq 80$ years old on these two landmark trials, as well as most randomized phase III lung cancer trials ${ }^{7}$, is likely multi-factorial. There are concerns with aggressive therapies causing excessive toxicity in an older cohort, elderly patients themselves choosing less-aggressive therapy, and the utility of therapy in patients with worsening performance status and medical comorbidites $7,8,18-21$. 
Given these concerns about aggressive chemoradiation in an elderly population, targeted agents and immunotherapy also play a role in therapy. While typically reserved for metastatic patients, recent reports indicate the incidence of molecular mutations appear congruent between early stage and late stage disease ${ }^{22-25}$. Additionally, there are ongoing clinical trials examining the impact of targeted agents and/or immunotherapy in the upfront non-metastatic setting ${ }^{26-28}$. Depending on these outcomes, these agents would be useful in an elderly population as their side effect profile is generally more favorable than traditional cytotoxic chemotherapy 29,30 .

Our series also confirmed known sociodemographic factors affecting OS in lung cancer patients in a geriatric population. Male sex was associated with lower OS in our series, similar to other large reviews of nationwide cancer mortality ${ }^{31}$. Stage IIIB (vs. IIIA) disease was associated with worse OS, which further validates modern staging criteria's impact on prognosis ${ }^{10}$. Patients with a Charlson-Deyo Score of 0 were associated with higher OS in our series, confirming that lack of medical comorbidities confers improved prognosis ${ }^{11,12}$ in elderly patients. Perhaps the most alarming finding in our series was that a significant portion of patients received no therapy at all. Other population-based efforts in lung cancer, mostly derived from Surveillance, Epidemiology, and End Results-Medicare databases, have estimated that between $34-44 \%$ of elderly patients (with varying definitions) do not receive tumor therapy ${ }^{32,33}$. Large database studies in other disease sites have also shown that elderly patients are less likely to receive therapy ${ }^{34}$. The higher rate of no therapy in our series is especially concerning as all patients in our series had health insurance which has been known to impact clinical outcomes ${ }^{35,36}$. Despite an insured population, socioeconomic barriers still seemed to influence receipt of care with black patients $23 \%$ more likely and patients living in a lower-educated census tract $20 \%$ more likely to not receive cancerdirected therapy, which has also been shown to effect general mortality rates in geriatric adults ${ }^{37}$. Our series also is the first to confirm in an elderly lung cancer population that patients treated at an academic center were more likely to receive CRT and be associated with improved OS, which previous series have suggested in younger populations ${ }^{38,39}$.

Our series has several strengths and limitations. The major strength of our series is our sample size; the largest study conducted of octogenarians and nonagenarians with stage III NSCLC. As the NCDB captures $70 \%$ of new cancer diagnoses, and includes many community centers, our elderly population is representative of a broad demographic, with treatment delivery and outcomes representative of the cancer community at large. However, like other studies from cancer registries, not all variables are captured, which may lead to an imbalance of the treatment groups, despite multivariable stratified analysis and propensityscore matched analysis. The benefit of receiving standard of care CRT persisted in all models, but confounding variables may still be present. Use of census tract estimates for socioeconomic variables introduces a possible fallacy, as we do not have the information of these variables at the individual patient level. Particularly important in elderly patients, treatment toxicity information is not captured in the NCDB, and we cannot comment on treatment toxicity in this series. CRT is known to be associated with fibrosis, esophagitis, myelosuppression, and cardiac damage $2,5,32,40$, and it is unclear if elderly patients can withstand concurrent therapy routinely. Lastly, the NCDB does not provide information on cause of death, and it is possible that the benefit of CRT seen on OS may be due to 
competing risks of mortality in this elderly population, although the Charlson-Deyo Comorbidity score and PSM testing were used to help account for competing risks of death.

\section{CONCLUSION}

In this analysis of the NCDB, over $62 \%$ of octogenarians and nonagenarians diagnosed with stage III NSCLC did not receive cancer-directed therapy. Patients that received the standard of care of concurrent chemoradiation had improved OS compared to those that did not. This benefit persisted when patients were matched for socioeconomic, demographic, tumor, and treatment facility characteristics. Alarmingly, despite an entirely insured population, we found that black race and living in a lower-educated county were associated with not receiving therapy. Patients seen at an academic center were more likely to receive some form of cancer-directed therapy. Further efforts to limit healthcare disparities in these elderly patients needs to be investigated.

\section{Supplementary Material}

Refer to Web version on PubMed Central for supplementary material.

\section{Acknowledgments}

Financial/Grant Support: This work was supported by the Biostatistics and Bioinformatics Shared Resource of Winship Cancer Institute of Emory University and National Cancer Institute at the National Institute of Health (Grant Number P30CA138292). This funding supported the statisticians who helped with study design, performed the analysis and helped with data interpretation. This funding did not have any role in the writing of the manuscript or the decision to submit it for publication. As the corresponding author, I have had full access to the data in the study and hold final responsibility for the decision to submit for publication. The content is solely the responsibility of the authors and does not necessarily represent the official views of the National Institutes of Health. The data used in the study are derived from a de-identified NCDB file. The American College of Surgeons and the Commission on Cancer have not verified and are not responsible for the analytic or statistical methodology employed, or the conclusions drawn from these data by the investigator.

\section{References}

1. Siegel RL, Miller KD, Jemal A. Cancer statistics, 2016. CA Cancer J Clin. 2016; 66:7-30. [PubMed: 26742998]

2. Curran WJ Jr, Paulus R, Langer CJ, et al. Sequential vs. concurrent chemoradiation for stage III non-small cell lung cancer: randomized phase III trial RTOG 9410. J Natl Cancer Inst. 2011; 103:1452-1460. [PubMed: 21903745]

3. Auperin A, Le Pechoux C, Pignon JP, et al. Concomitant radio-chemotherapy based on platin compounds in patients with locally advanced non-small cell lung cancer (NSCLC): a meta-analysis of individual data from 1764 patients. Ann Oncol. 2006; 17:473-483. [PubMed: 16500915]

4. Zatloukal P, Petruzelka L, Zemanova M, et al. Concurrent versus sequential chemoradiotherapy with cisplatin and vinorelbine in locally advanced non-small cell lung cancer: a randomized study. Lung Cancer. 2004; 46:87-98. [PubMed: 15364136]

5. Bradley JD, Paulus R, Komaki R, et al. Standard-dose versus high-dose conformal radiotherapy with concurrent and consolidation carboplatin plus paclitaxel with or without cetuximab for patients with stage IIIA or IIIB non-small-cell lung cancer (RTOG 0617): a randomised, two-by-two factorial phase 3 study. Lancet Oncol. 2015; 16:187-199. [PubMed: 25601342]

6. Hutchins LF, Unger JM, Crowley JJ, Coltman CA Jr, Albain KS. Underrepresentation of patients 65 years of age or older in cancer-treatment trials. N Engl J Med. 1999; 341:2061-2067. [PubMed: 10615079] 
7. Sacher AG, Le LW, Leighl NB, Coate LE. Elderly patients with advanced NSCLC in phase III clinical trials: are the elderly excluded from practice-changing trials in advanced NSCLC? J Thorac Oncol. 2013; 8:366-368. [PubMed: 23407560]

8. Pallis AG, Gridelli C, Wedding U, et al. Management of elderly patients with NSCLC; updated expert's opinion paper: EORTC Elderly Task Force, Lung Cancer Group and International Society for Geriatric Oncology. Ann Oncol. 2014; 25:1270-1283. [PubMed: 24638905]

9. Kent EE, Malinoff R, Rozjabek HM, et al. Revisiting the Surveillance Epidemiology and End Results Cancer Registry and Medicare Health Outcomes Survey (SEER-MHOS) Linked Data Resource for Patient-Reported Outcomes Research in Older Adults with Cancer. J Am Geriatr Soc. 2016; 64:186-192. [PubMed: 26782871]

10. Edge, SB. AJCC cancer staging manual. 7. New York: Springer; 2010. American Joint Committee on Cancer.

11. Deyo RA, Cherkin DC, Ciol MA. Adapting a clinical comorbidity index for use with ICD-9-CM administrative databases. J Clin Epidemiol. 1992; 45:613-619. [PubMed: 1607900]

12. Charlson ME, Pompei P, Ales KL, MacKenzie CR. A new method of classifying prognostic comorbidity in longitudinal studies: development and validation. J Chronic Dis. 1987; 40:373-383. [PubMed: 3558716]

13. Nickleach DLY, Shrewsberry A, et al. SAS® Macros to Conduct Common Biostatistical Analyses and Generate Reports. SESUG 2013: The Proceeding of the SouthEast SAS User Group. 2013

14. LS P. Reducing bias in a propensity score matched pair sample using greedy matching techniques. SAS SUGI. 2001; 26:214-226.

15. Austin PC, Grootendorst P, Anderson GM. A comparison of the ability of different propensity score models to balance measured variables between treated and untreated subjects: a Monte Carlo study. Stat Med. 2007; 26:734-753. [PubMed: 16708349]

16. Gustafson P. On robustness and model flexibility in survival analysis: transformed hazard models and average effects. Biometrics. 2007; 63:69-77. [PubMed: 17447931]

17. DY Lin LW. The Robust Inference for the Cox Proportional Hazards Model. Journal of the American Statistical Association. 1989; 84:1074-1078.

18. Hurria A, Levit LA, Dale W, et al. Improving the Evidence Base for Treating Older Adults With Cancer: American Society of Clinical Oncology Statement. J Clin Oncol. 2015; 33:3826-3833. [PubMed: 26195697]

19. Jatoi A, Hillman S, Stella P, et al. Should elderly non-small-cell lung cancer patients be offered elderly-specific trials? Results of a pooled analysis from the North Central Cancer Treatment Group. J Clin Oncol. 2005; 23:9113-9119. [PubMed: 16361618]

20. Maione P, Rossi A, Sacco PC, et al. Treating advanced non-small cell lung cancer in the elderly. Ther Adv Med Oncol. 2010; 2:251-260. [PubMed: 21789138]

21. Losanno T, Gridelli C. Recent advances in targeted advanced lung cancer therapy in the elderly. Expert Rev Anticancer Ther. 2017

22. Cancer Genome Atlas Research N. Comprehensive molecular profiling of lung adenocarcinoma. Nature. 2014; 511:543-550. [PubMed: 25079552]

23. Sholl LM, Aisner DL, Varella-Garcia M, et al. Multi-institutional Oncogenic Driver Mutation Analysis in Lung Adenocarcinoma: The Lung Cancer Mutation Consortium Experience. J Thorac Oncol. 2015; 10:768-777. [PubMed: 25738220]

24. Cassidy RJ, Zhang X, Patel PR, et al. Next-generation sequencing and clinical outcomes of patients with lung adenocarcinoma treated with stereotactic body radiotherapy. Cancer. 2017

25. Lin MW, Wu CT, Shih JY, Chang YL, Yang PC. Clinicopathologic characteristics and prognostic significance of EGFR and p53 mutations in surgically resected lung adenocarcinomas $</=2 \mathrm{~cm}$ in maximal dimension. J Surg Oncol. 2014; 110:99-106. [PubMed: 24788590]

26. (NCI) NCI. Genetic Testing in Screening Patients With Stage IB-IIIA Non-Small Cell Lung Cancer That Has Been or Will Be Removed by Surgery (The ALCHEMIST Screening Trial). Available from URL: https://clinicaltrials.gov/ct2/show/NCT02194738?term=alchemist+trial\&rank=2

27. ZuhGauangYing. [accessed 8/1/2017] Radiotherapy Combined With Iressa for EGFR Mutation Positive Patients With Locally Advanced Non-small Cell Lung Cancer (NSCLC) - NCT01391260. Available from URL: https://clinicaltrials.gov/ct2/show/NCT01391260 
28. Institute NC. Erlotinib Hydrochloride or Crizotinib and Chemoradiation Therapy in Treating Patients With Stage III Non-small Cell Lung Cancer - NCT018224962017.

29. Simone CB 2nd, Burri SH, Heinzerling JH. Novel radiotherapy approaches for lung cancer: combining radiation therapy with targeted and immunotherapies. Transl Lung Cancer Res. 2015; 4:545-552. [PubMed: 26629423]

30. Mok TS, Wu YL, Thongprasert S, et al. Gefitinib or carboplatin-paclitaxel in pulmonary adenocarcinoma. N Engl J Med. 2009; 361:947-957. [PubMed: 19692680]

31. Cook MB, McGlynn KA, Devesa SS, Freedman ND, Anderson WF. Sex disparities in cancer mortality and survival. Cancer Epidemiol Biomarkers Prev. 2011; 20:1629-1637. [PubMed: 21750167]

32. Owonikoko TK, Ragin CC, Belani CP, et al. Lung cancer in elderly patients: an analysis of the surveillance, epidemiology, and end results database. J Clin Oncol. 2007; 25:5570-5577. [PubMed: 18065729]

33. Davidoff AJ, Gardner JF, Seal B, Edelman MJ. Population-based estimates of survival benefit associated with combined modality therapy in elderly patients with locally advanced non-small cell lung cancer. J Thorac Oncol. 2011; 6:934-941. [PubMed: 21372743]

34. Cassidy RJ, Switchenko JM, Cheng E, et al. Health care disparities among octogenarians and nonagenarians with stage II and III rectal cancer. Cancer. 2017

35. Ward E, Halpern M, Schrag N, et al. Association of insurance with cancer care utilization and outcomes. CA Cancer J Clin. 2008; 58:9-31. [PubMed: 18096863]

36. Slatore CG, Au DH, Gould MK. American Thoracic Society Disparities in Healthcare G. An official American Thoracic Society systematic review: insurance status and disparities in lung cancer practices and outcomes. Am J Respir Crit Care Med. 2010; 182:1195-1205. [PubMed: 21041563]

37. Huisman M, Read S, Towriss CA, Deeg DJ, Grundy E. Socioeconomic inequalities in mortality rates in old age in the World Health Organization Europe region. Epidemiol Rev. 2013; 35:84-97. [PubMed: 23382476]

38. Samson P, Patel A, Crabtree TD, et al. Multidisciplinary Treatment for Stage IIIA Non-Small Cell Lung Cancer: Does Institution Type Matter? Ann Thorac Surg. 2015; 100:1773-1779. [PubMed: 26228601]

39. Parsons HM, Harlan LC, Stevens JL, Ullmann CD. Treatment of small cell lung cancer in academic and community settings: factors associated with receiving standard therapy and survival. Cancer J. 2014; 20:97-104. [PubMed: 24667952]

40. Prabhu RS, Cassidy RJ, Landry JC. Radiation therapy and neutropenia. Curr Probl Cancer. 2015; 39:292-296. [PubMed: 26293689] 


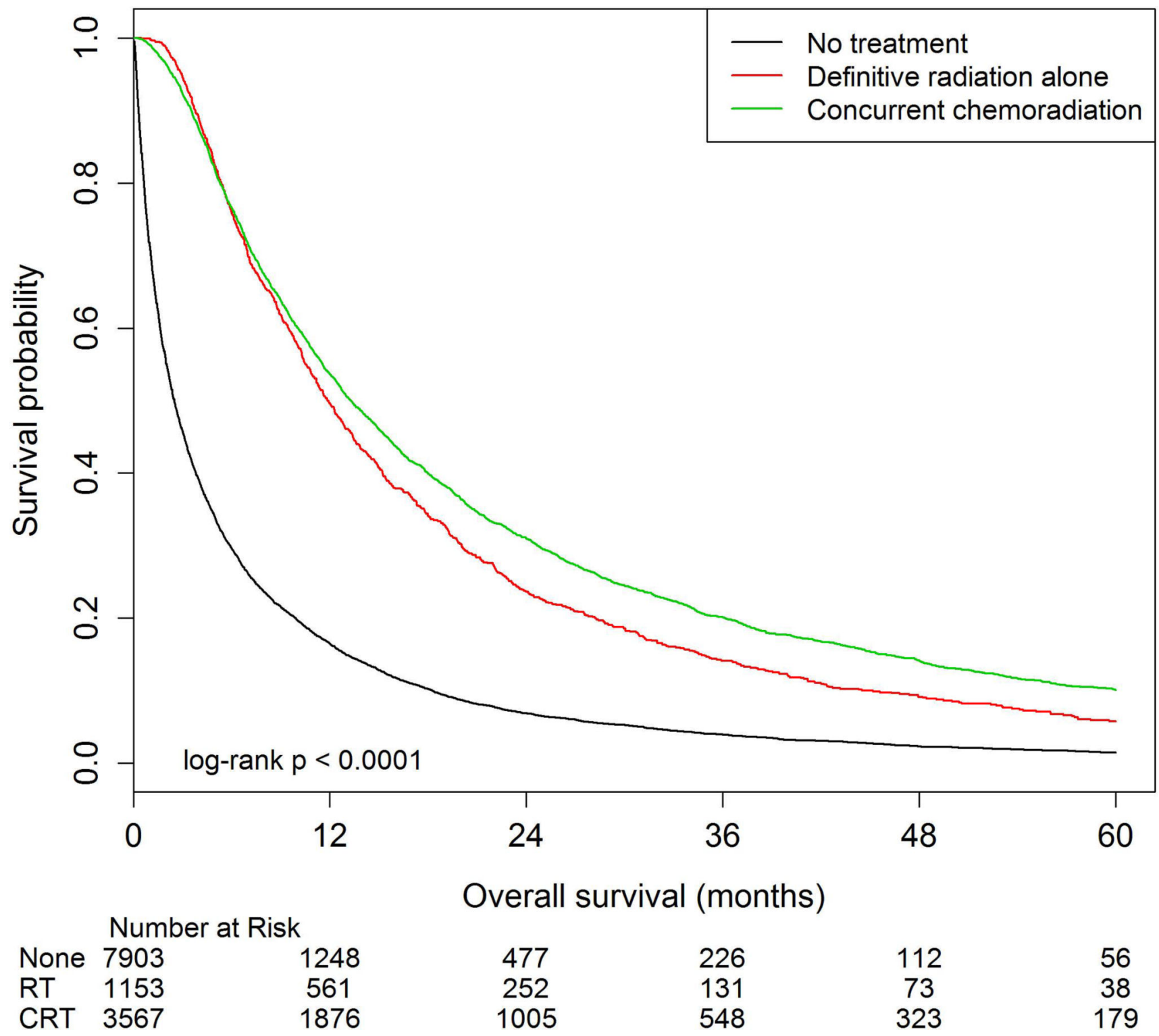

Figure 1. Overall survival among patients 80 years and older with stage III lung cancer treated with concurrent chemoradiation, definitive radiation alone, and those who did not receive therapy

Overall survival (OS) was calculated as time from diagnosis to time to death. The number at risk is delineated above the $\mathrm{x}$ axis at each time point. Kaplan-Meier estimates for median OS were 13.3 months (95\%CI: 12.7-14.0), 11.9 months (95\%CI: 11.2-12.7), and 2.5 months (95\% CI: 2.4-2.6) for patients treated with concurrent chemoradiation, definitive radiation alone, and no treatment respectively ( $\mathrm{p}<0.01)$. 5-year OS estimates were compared using log-rank tests, with rates of $10.1 \%$ (95\%CI: 9.0-11.3), 5.7\% (95\%CI: 4.3-7.5), and 1.5\% (95\%CI: 1.2-1.8) for patients treated with concurrent chemoradiation, definitive radiation alone, and no treatment respectively $(\mathrm{p}<0.01)$. 


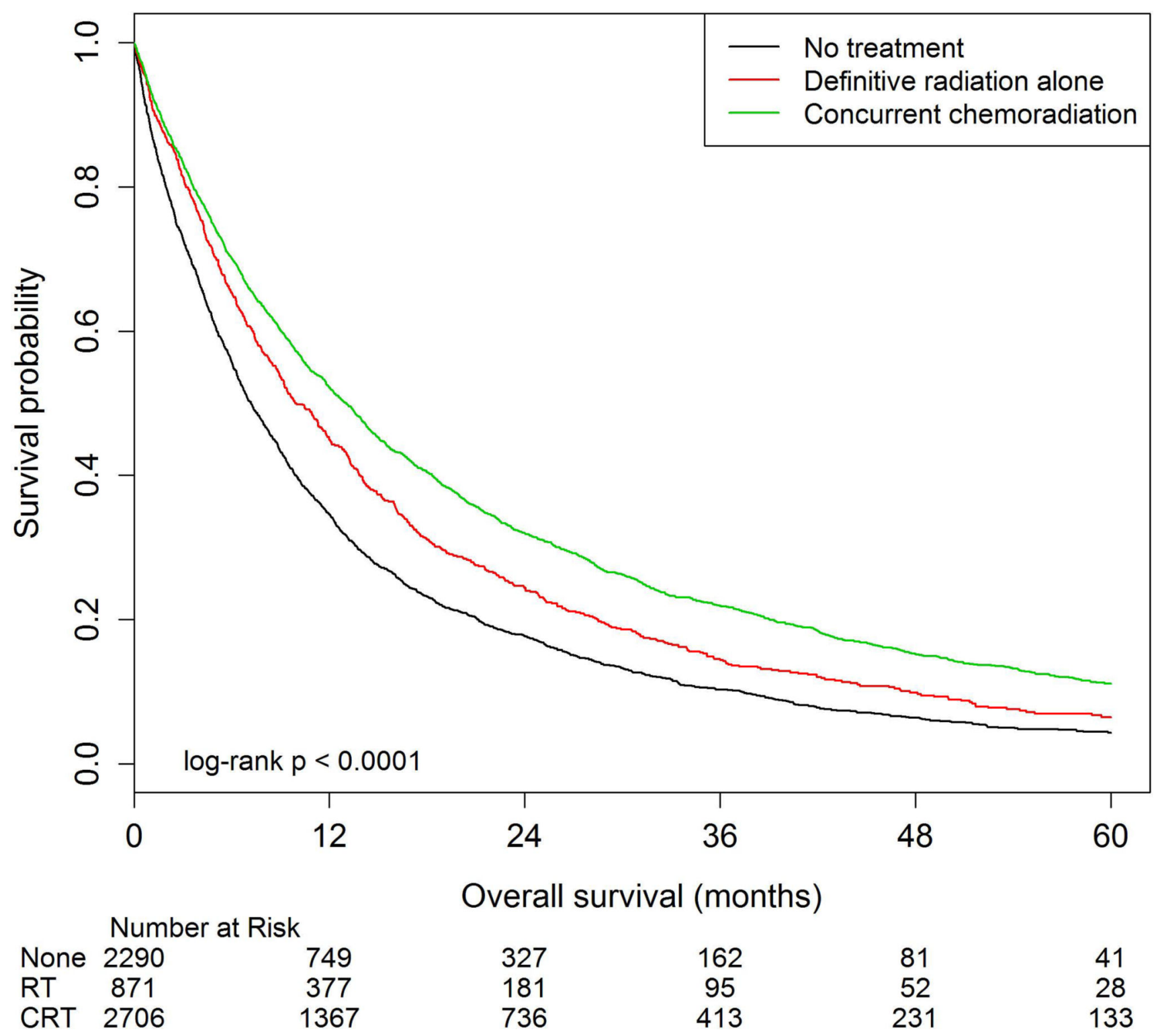

Figure 2. Overall survival among patients 80 years and older with stage III lung cancer treated with concurrent chemoradiation, definitive radiation alone, and those who did not receive therapy, excluding patients who died within six months

Overall survival (OS) was calculated as time from diagnosis to time to death. Patients who died within 6 months of diagnosis were excluded from analysis. The number at risk is delineated above the $\mathrm{x}$ axis at each time point. Kaplan-Meier estimates for median OS were 13.2 months (95\%CI: 12.7-14), 9.6 months (95\%CI: 9.0-10.2), and 7.2 months (95\%CI: 6.1-8.3) for patients treated with concurrent chemoradiation, definitive radiation alone, and no treatment respectively ( $\mathrm{p}<0.01)$. 5-year OS estimates were compared using log-rank tests, with rates of $11.2 \%$ (95\%CI: 9.7-12.7), 6.5\% (95\%CI: 4.7-8.6), and 4.3\% (95\%CI: 3.3-5.5) for patients treated with concurrent chemoradiation, definitive radiation alone, and no treatment respectively $(\mathrm{p}<0.01)$. 


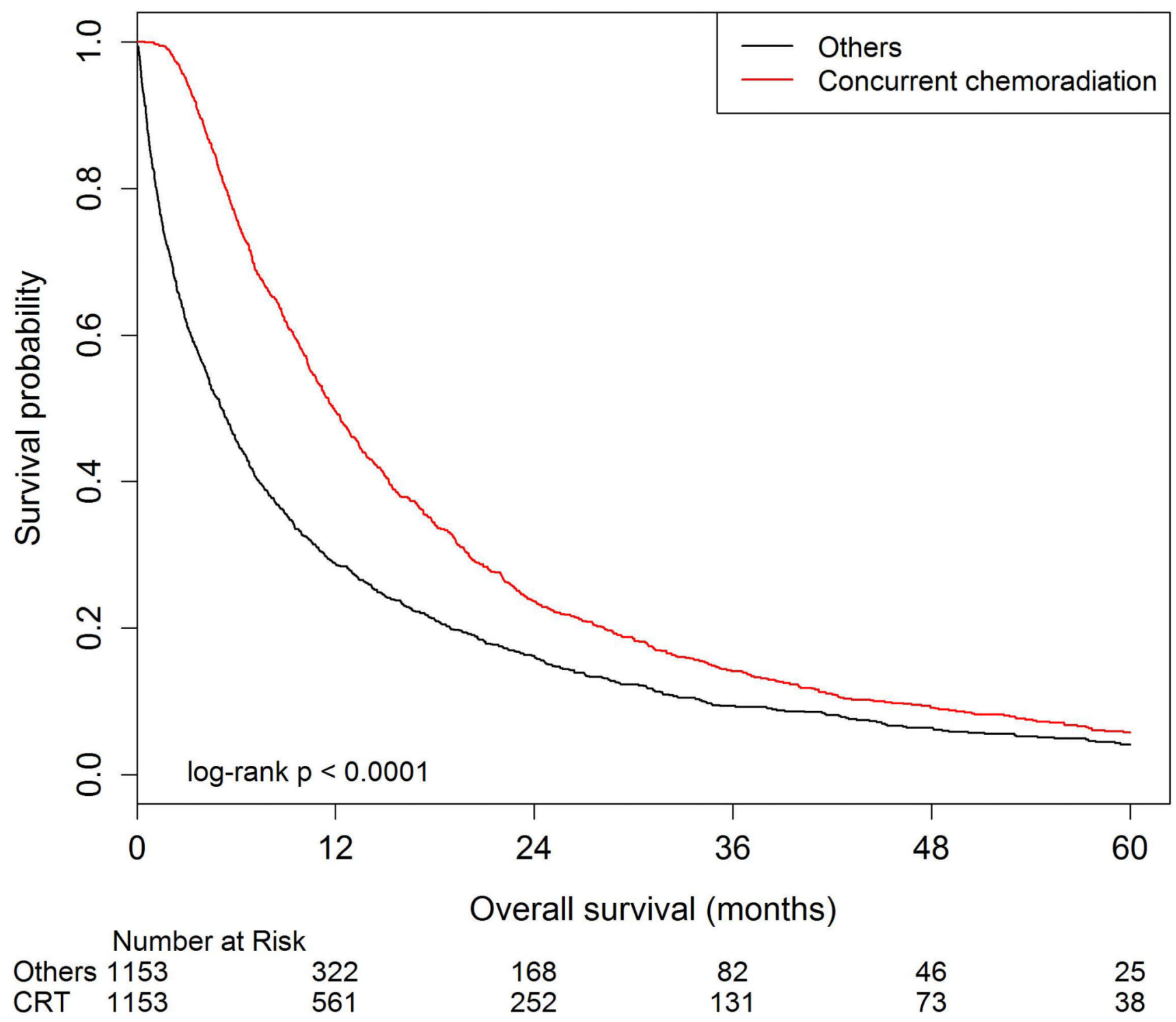

Figure 3. Overall survival amongst patients 80 years and older with stage III lung cancer among patients receiving concurrent chemoradiation versus other treatment modalities identified by the propensity score matching method

Overall survival estimates were generated from a balanced propensity score matched (PSM)

cohort using a 5-1-digit matching algorithm to match patients 1:1. After matching, PSM analysis identified 933 patients in each group. The number at risk is delineated above thexaxis at each time point. These cohorts were balanced for patient, tumor, and other treatment characteristics based on standard difference values of all greater than 0.10 . Kaplan-Meier estimates for overall survival show a median survival of 14.9 months (95\% CI 13.5-15.9) for patients receiving chemoradiation compared to 12.2 months (95\%CI 11.213.4) for other treatment modalities $(\mathrm{p}<0.01)$. The 3-year OS estimates were $21.5 \%$ (95\%CI: 18.7-24.3) for patients receiving chemoradiation compared to $15.8 \%$ (95\% CI: 13.4-18.3) for other treatment modalities $(\mathrm{p}<0.01)$. 

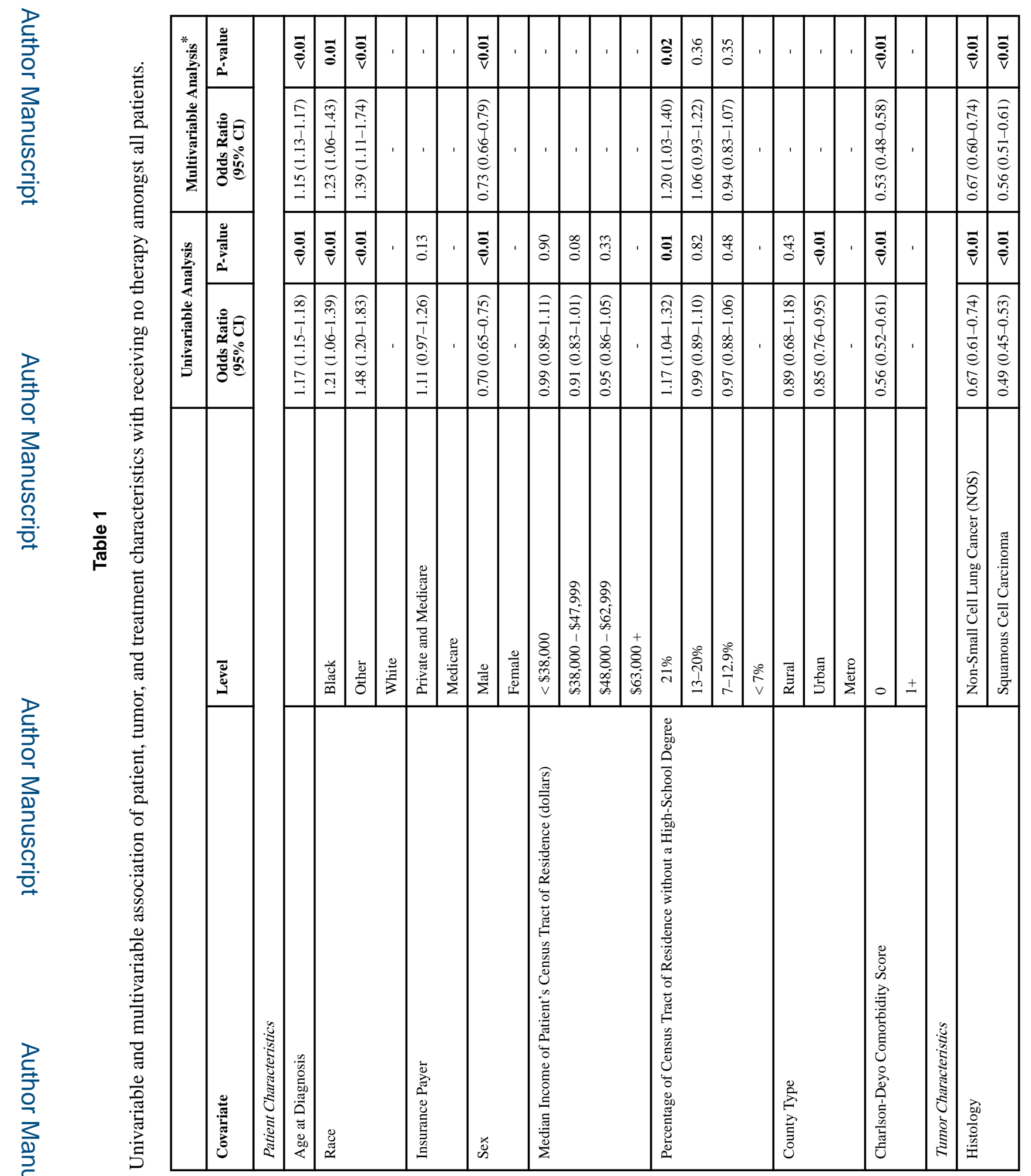

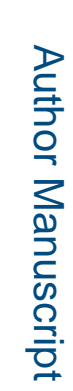

Cancer. Author manuscript; available in PMC 2019 February 15. 


$$
\text { ב্ }
$$

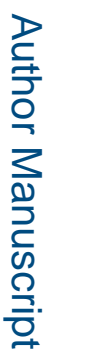

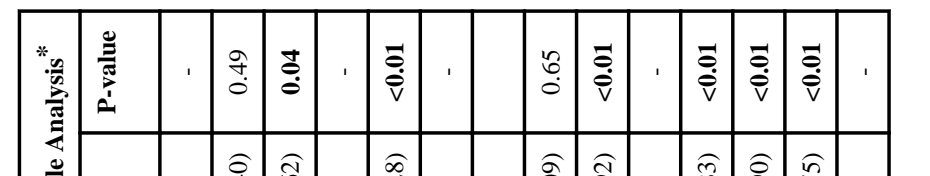

를

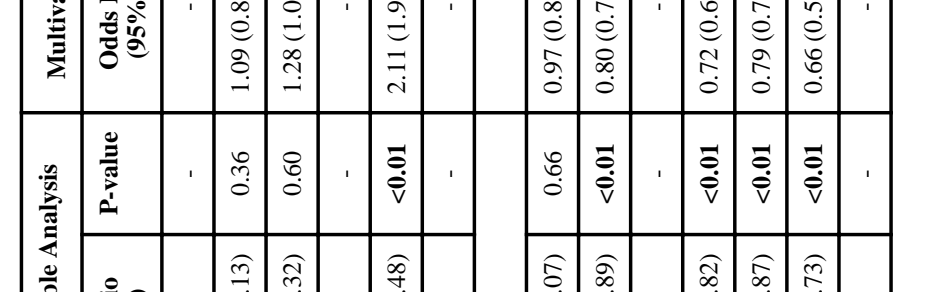

t

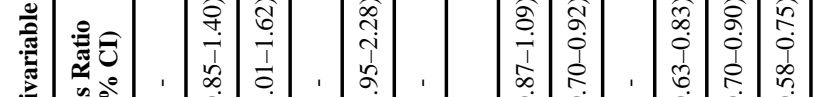
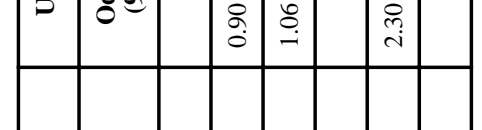

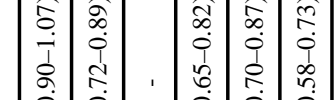

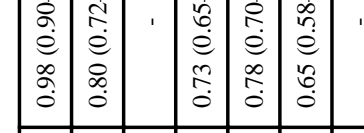

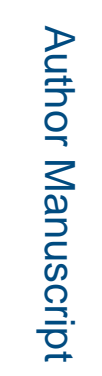

.
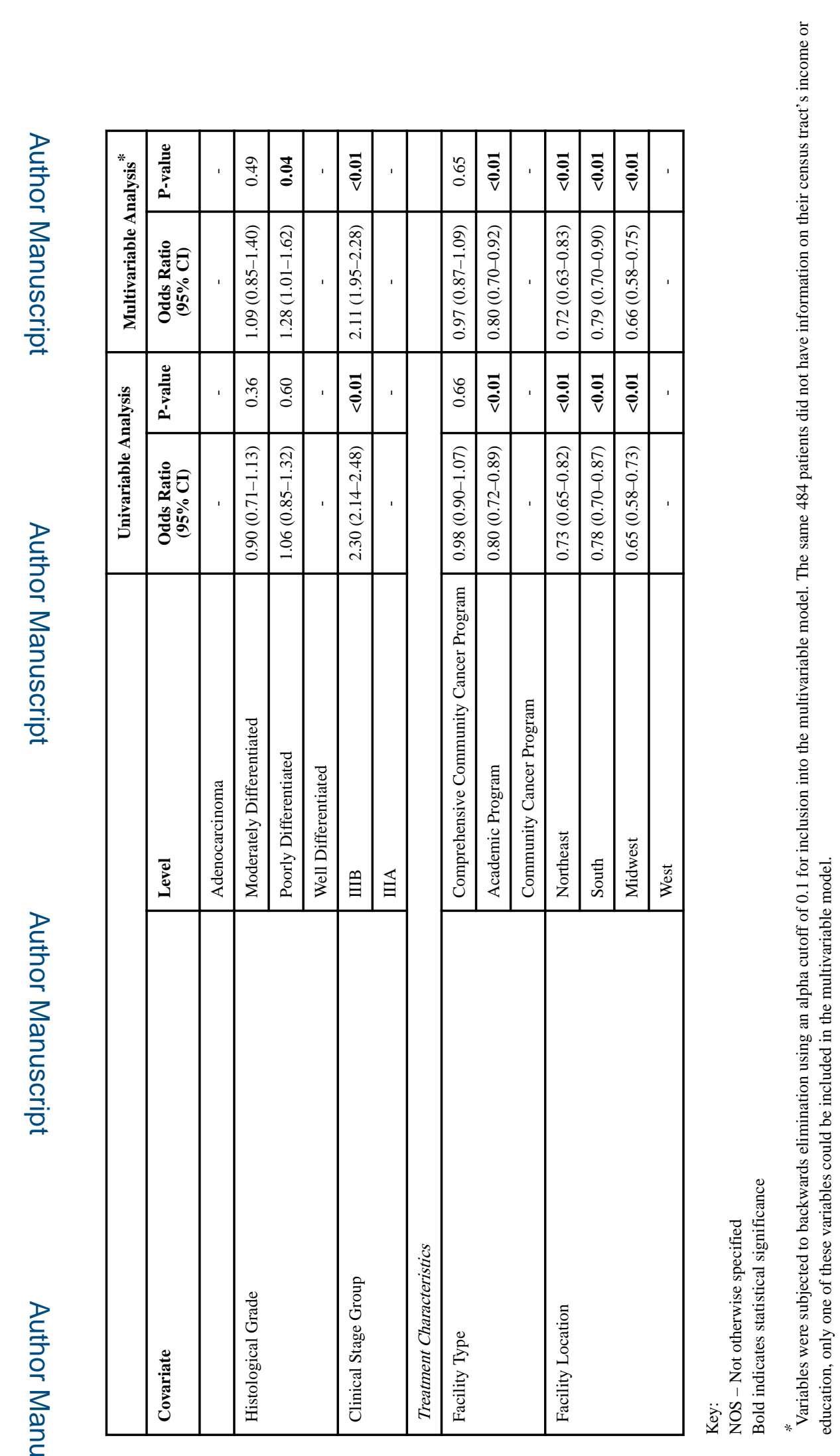

Cancer. Author manuscript; available in PMC 2019 February 15. 


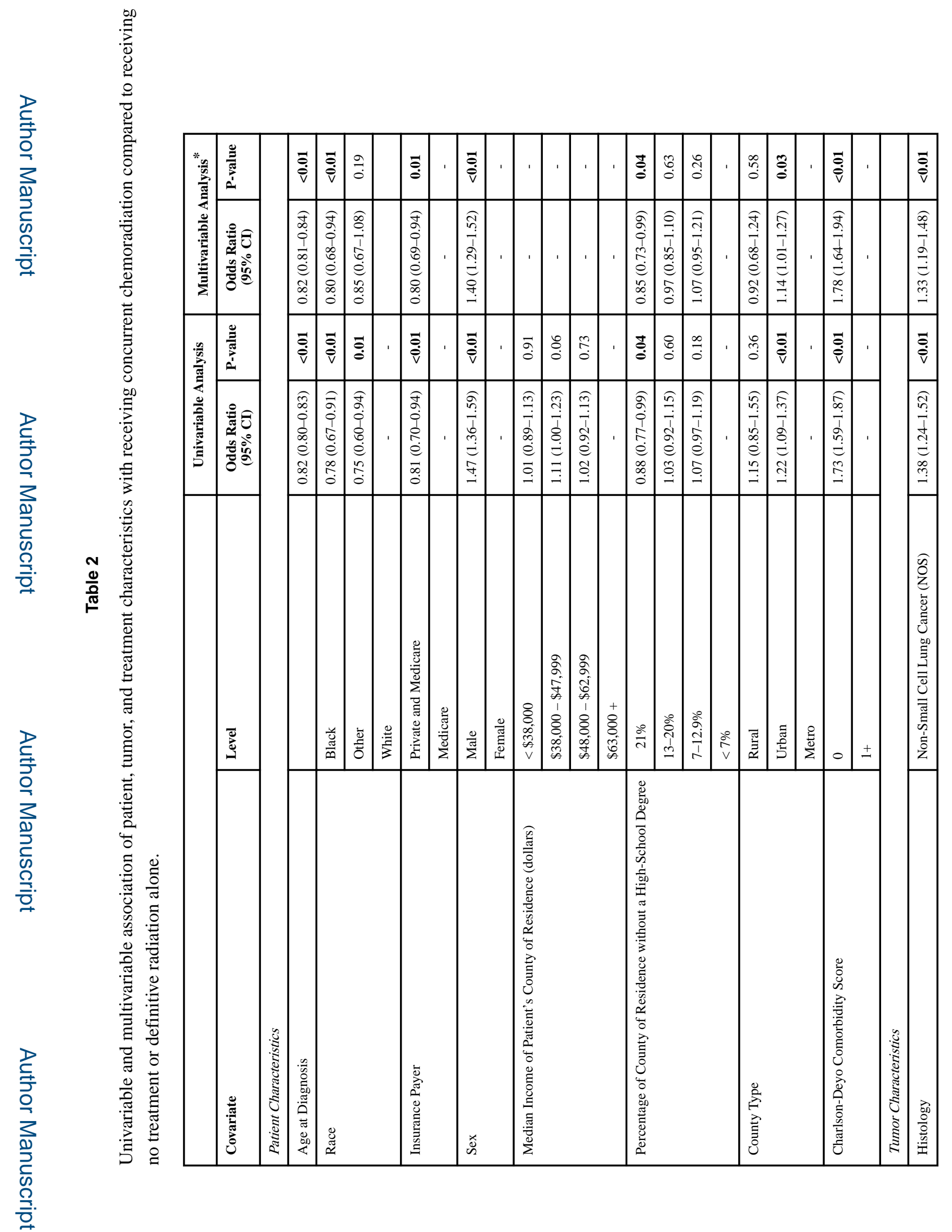

Cancer. Author manuscript; available in PMC 2019 February 15. 


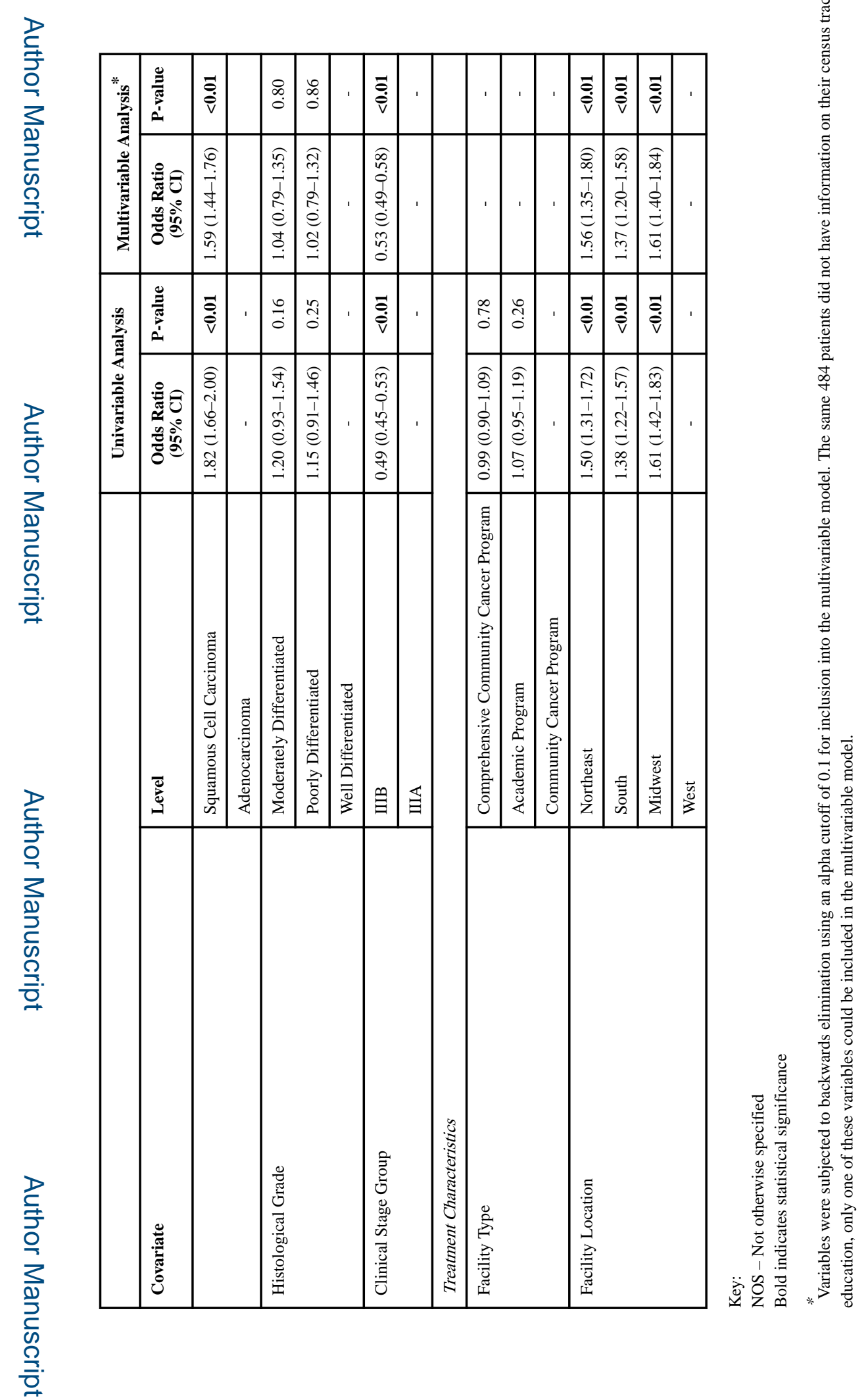

Cancer. Author manuscript; available in PMC 2019 February 15. 


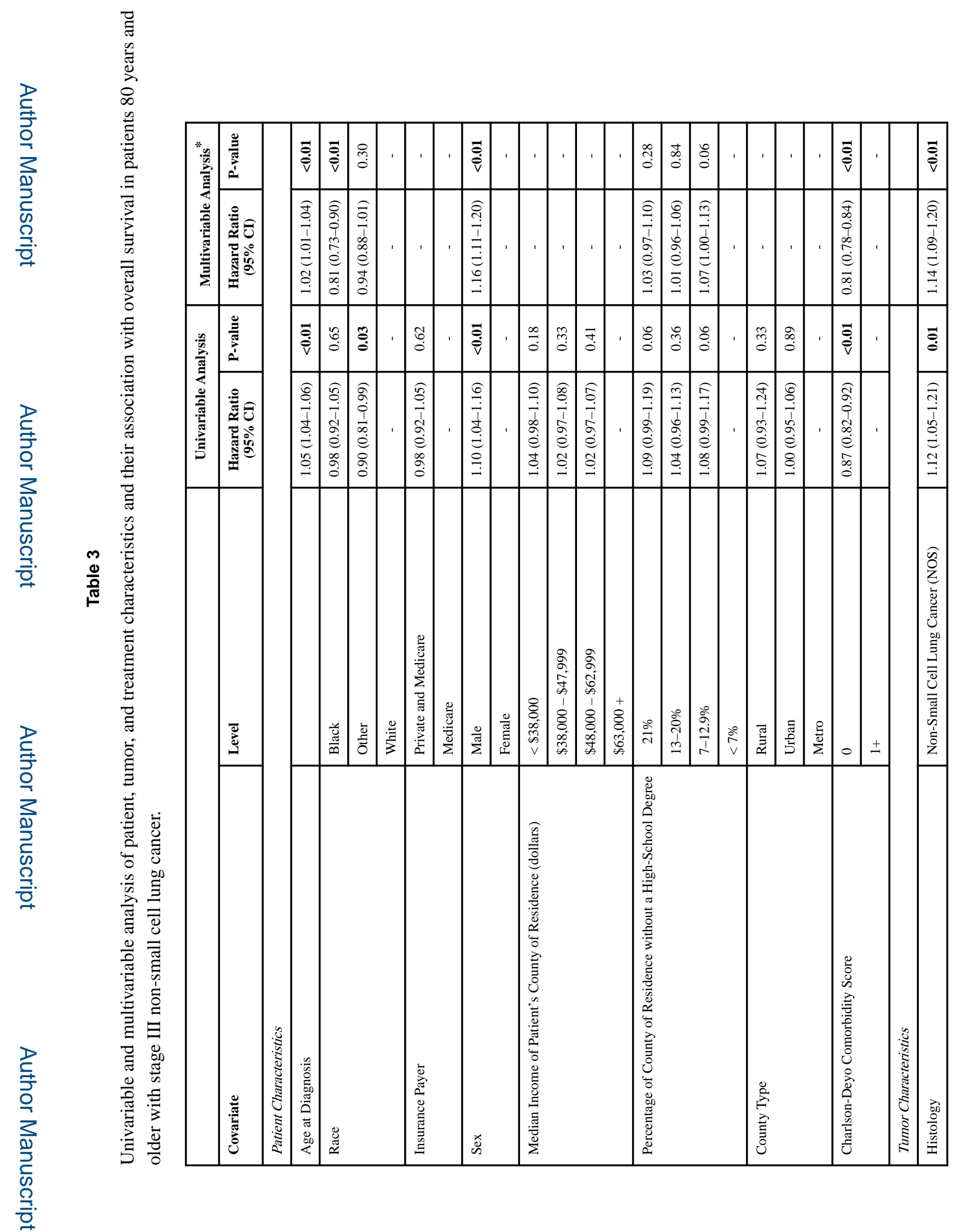

Cancer. Author manuscript; available in PMC 2019 February 15. 


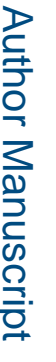

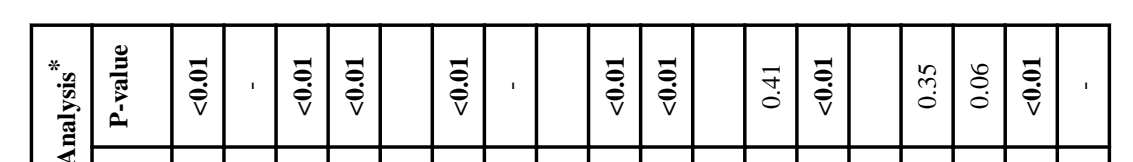

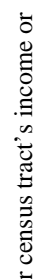

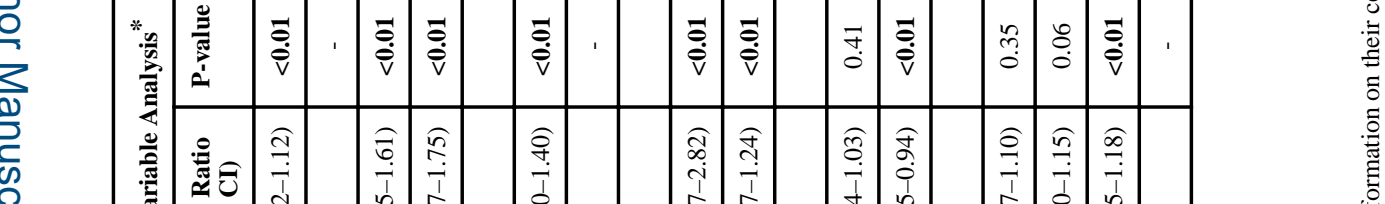

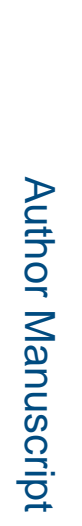

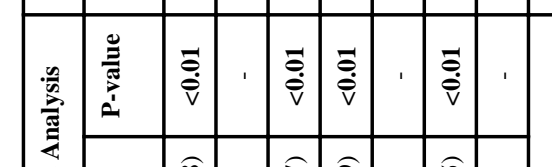

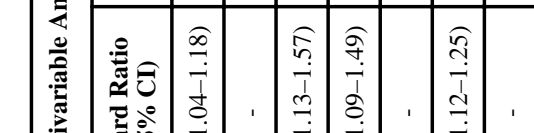

\begin{tabular}{|c|c|c|c|c|c|c|c|c|c|c|c|c|c|c|c|c|c|c|}
\hline & 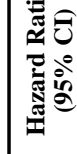 & 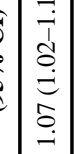 & $\cdot$ & 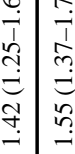 & & 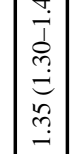 & . & & 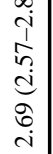 & 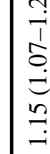 & & & & $\mid \begin{array}{c}0 \\
1 \\
0 \\
0 \\
0 \\
e \\
0 \\
0 \\
0 \\
0\end{array}$ & & $\mid \begin{array}{l}\dot{T} \\
\hat{\alpha} \\
\hat{e} \\
\tilde{o} \\
\dot{g}\end{array}$ & 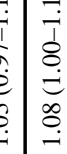 & 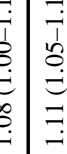 \\
\hline & $\cong$ & $1=$ & & $=0$ & & $=$ & & & & & & & & & & & & \\
\hline
\end{tabular}

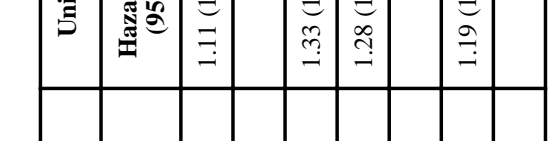

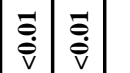

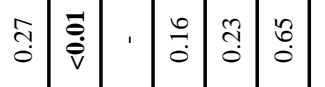

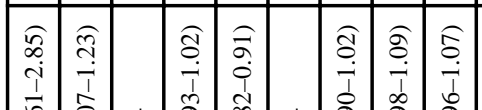

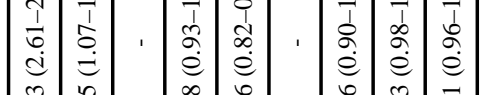

\section{i}

奠
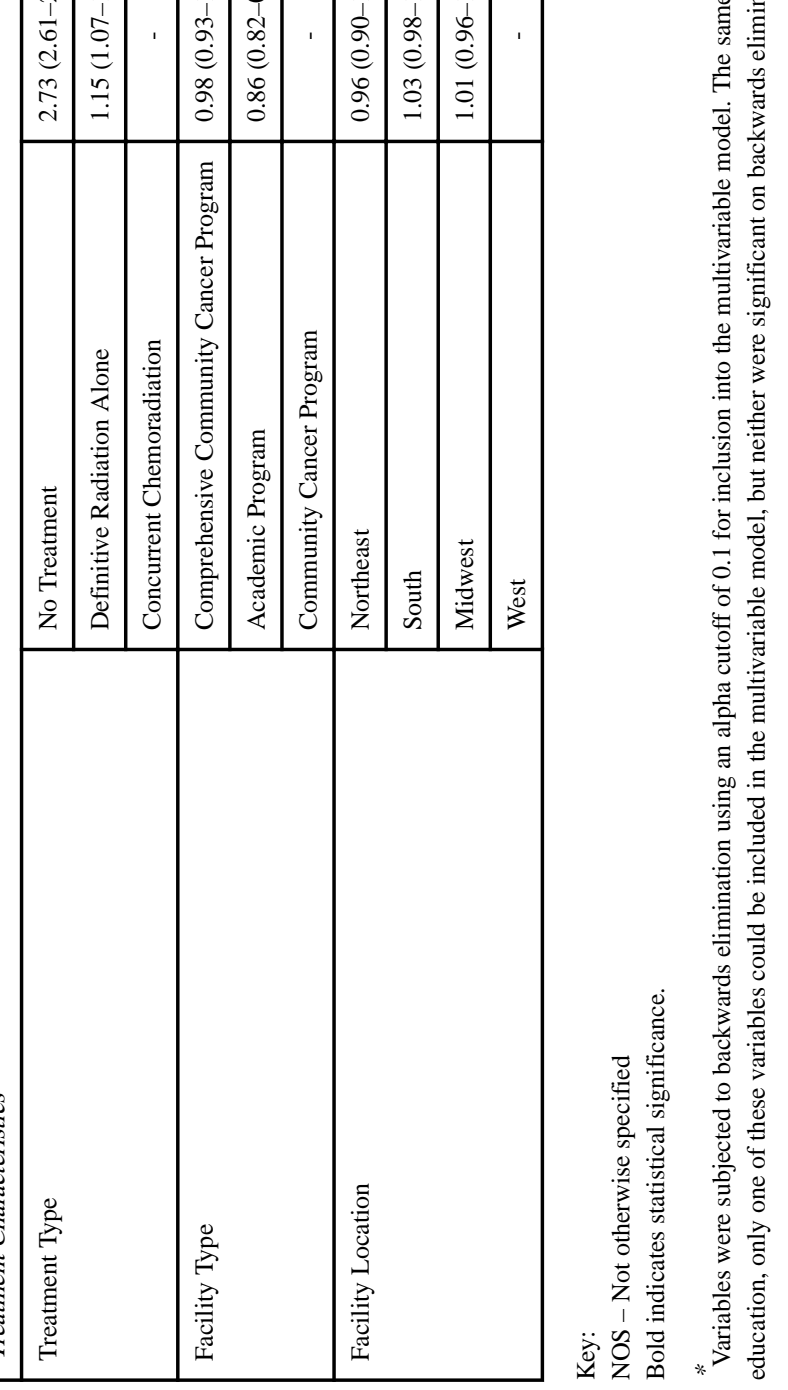



를

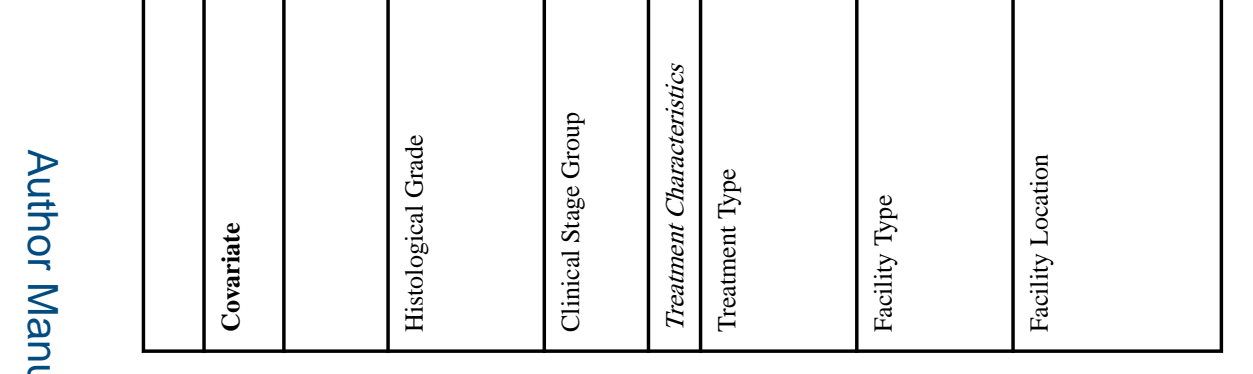
乙吊* 